Supporting Information for

\title{
Bifunctional Electrocatalytic Activity of Bis(iminothiolato)nickel Monolayer for Overall Water Splitting
}

Xiaohan Song, Junru Wang, Siyun Qi, Yingcai Fan, Weifeng Li, Mingwen Zhao* School of Physics and State Key Laboratory of Crystal Materials, Shandong University, Jinan 250100, China

Corresponding author, E-mail: zmw@sdu.edu.cn 
Table S1. Zero-pint energy correction $\left(\mathrm{E}_{\mathrm{ZPE}}\right)$ of the adsorbates considered in this work.

\begin{tabular}{llll}
\hline NiIT & $\mathbf{E}_{\mathbf{Z P E}}(\mathrm{eV})$ & FeIT & $\mathbf{E}_{\mathbf{Z P E}}(\mathbf{e V})$ \\
\hline $\mathbf{O H} *$ & 0.39 & $\mathbf{O H}^{*}$ & 0.4 \\
$\mathbf{O *}$ & 0.08 & $\mathbf{O *}$ & 0.08 \\
$\mathbf{O O H}^{*}$ & 0.47 & $\mathbf{O O H} *$ & 0.46 \\
$\mathbf{H}^{*}$ & 0.34 & $\mathbf{H}^{*}$ & 0.33 \\
\hline
\end{tabular}


(a)
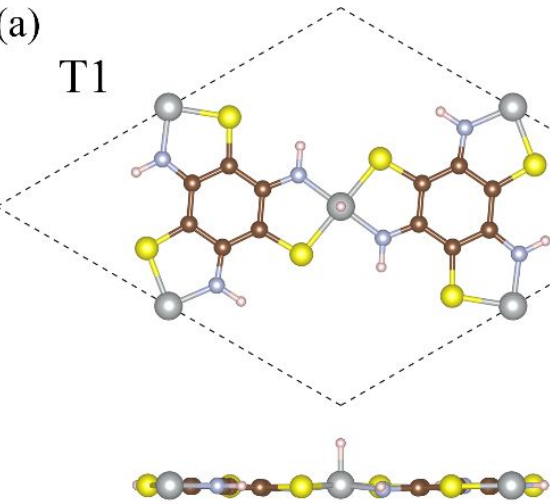

(c)

(c) $\mathrm{T} 4$

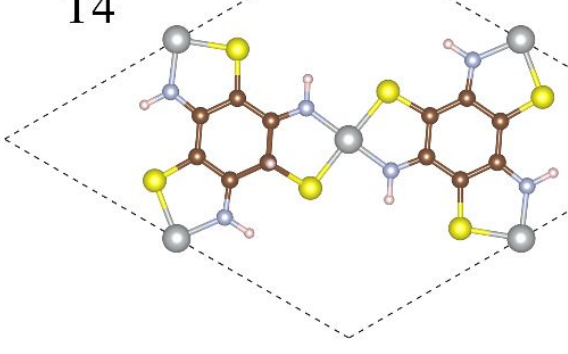

$\cos =0-0-0-0,0=0$ (b)

\section{T3}
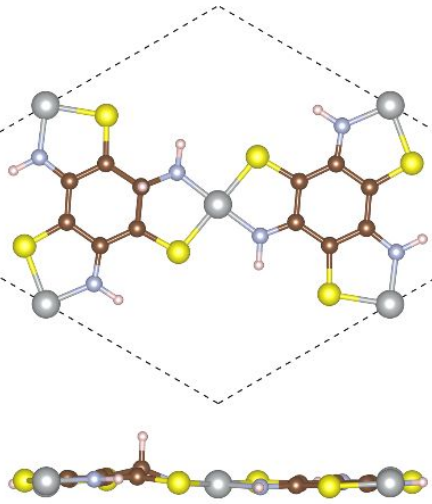

(d)

T5

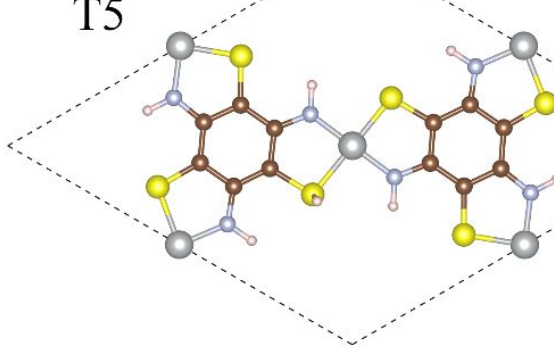

$\cos 0-0-0-0-0=0$

Figure S1. The HER intermediate $\left(\mathrm{H}^{*}\right)$ at (a) T1, (b) T3, (c) T4 and (d) T5 sites of the NiIT monolayer. 


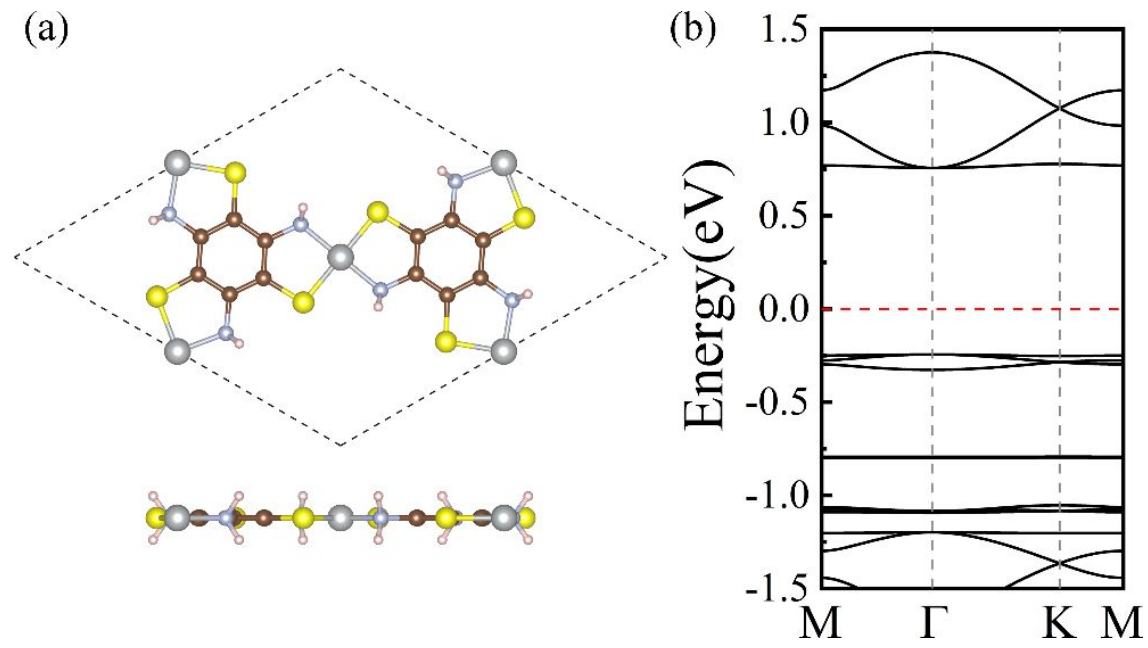

Figure S2. (a) Top (upper) and side (bottom) views of optimized structure of the NiAT monolayer where the $\mathrm{N}$ atom is terminated by two $\mathrm{H}$ atoms. The dashed diamond marks the primitive cell of the monolayer. (b) Band structure of NiAT monolayer. The energy at the Fermi level was set to zero. 
$\mathrm{OH}^{*}$

$\mathrm{O}^{*}$

$\mathrm{OOH}^{*}$

(a) $\mathrm{T} 1$
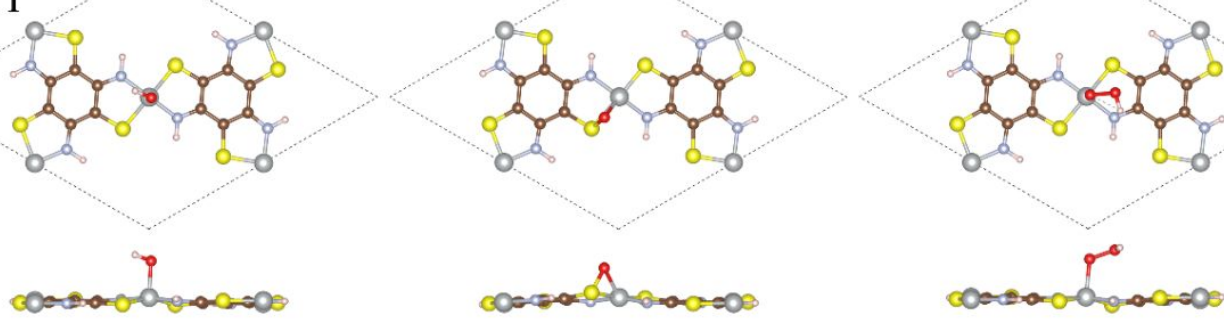

(b) $\mathrm{T}$
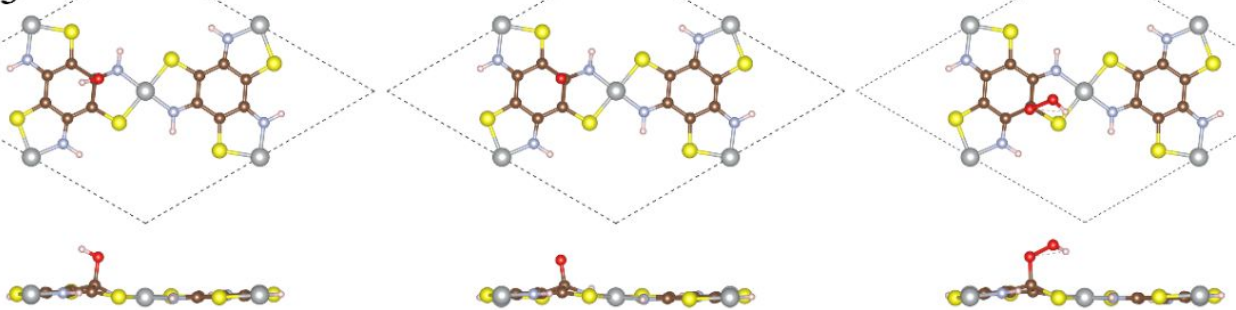

(c) $\mathrm{T5}$
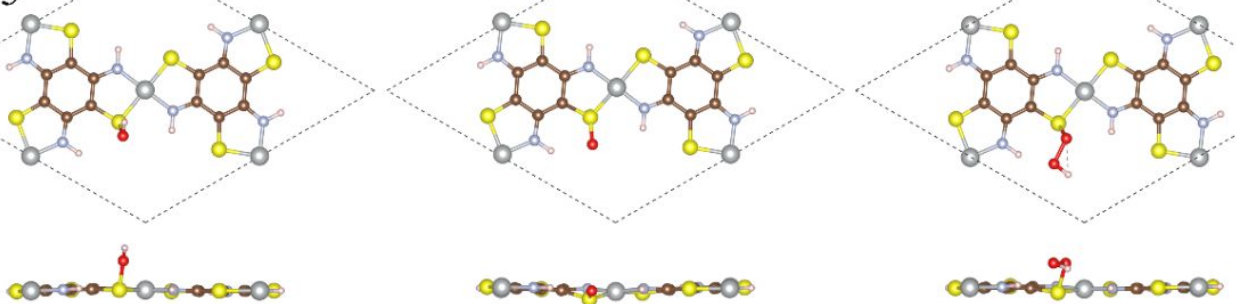

Figure S3. The OER intermediates $\left(\mathrm{OH}^{*}, \mathrm{O}^{*}\right.$, and $\left.\mathrm{OOH}^{*}\right)$ at $(\mathrm{a}) \mathrm{T} 1,(\mathrm{~b}) \mathrm{T} 3$ and (c)T5 sites of the NiIT monolayer. 


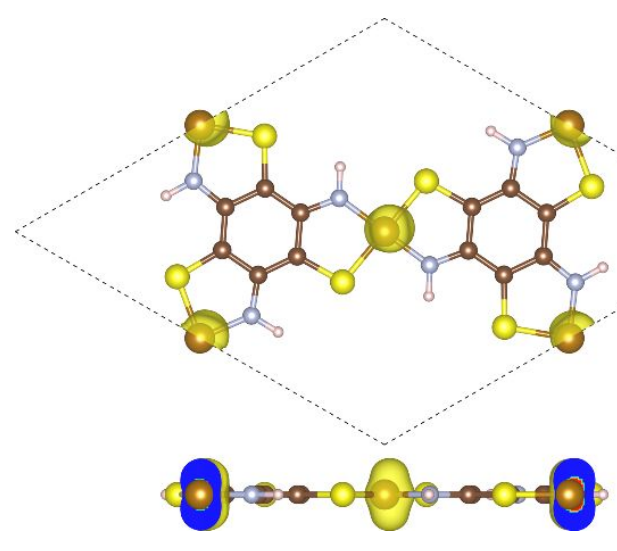

Figure S4. Top (upper) and side (bottom) views of the spin density isosurfaces of the FeIT monolayer.

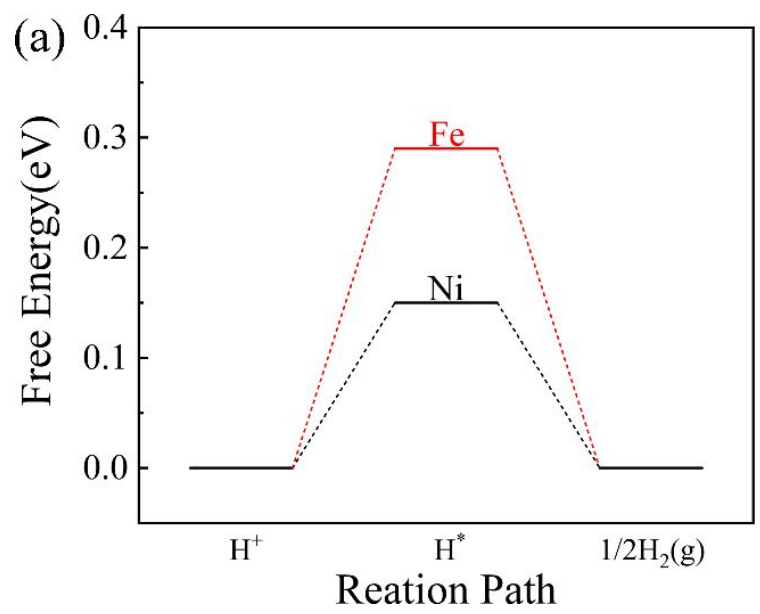

(b)

Figure S5. (a)The calculated HER Gibbs free energy diagram at T2 site of NiIT and FeIT monolayer at zero potential $(U=0)$. (b)The corresponding structure configurations of HER intermediates of FeIT monolayer. 


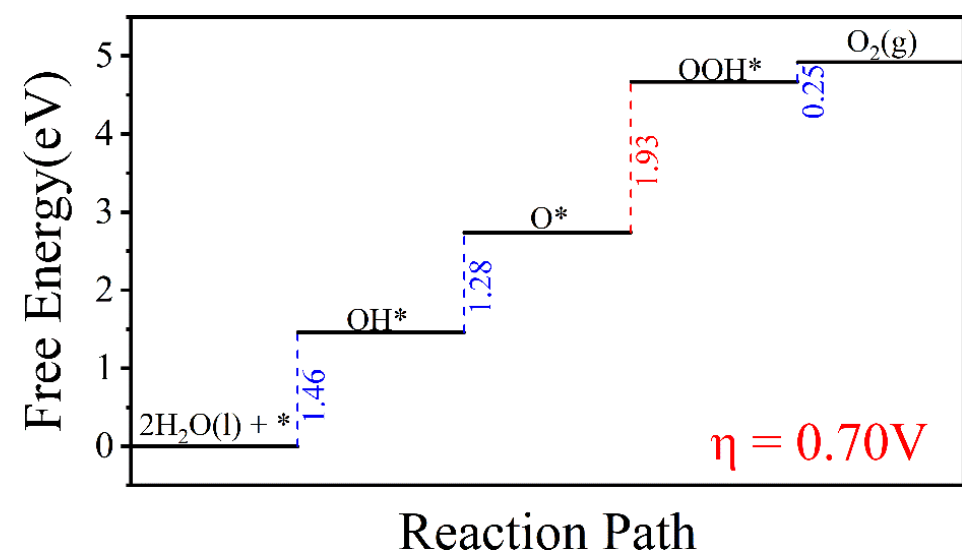

Figure S6. The calculated OER Gibbs free energy diagram at T4 site of FeIT monolayer at zero potential, where the elementary reaction with $\Delta \mathrm{G}$ in red represents the potential-determining step.

(a)

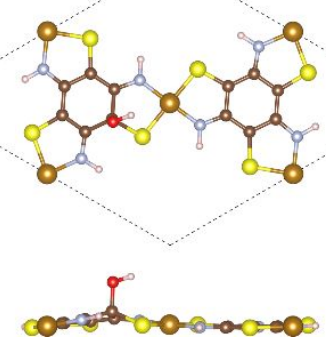

(b)

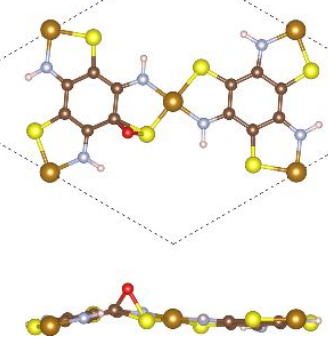

(c)

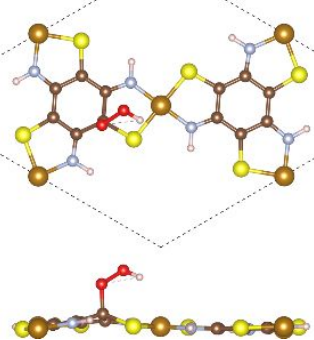

Figure S7. (a-c) The structures of the OER intermediates $\mathrm{OH}^{*}, \mathrm{O}^{*}$ and $\mathrm{OOH}^{*}$ at the T4 site of FeIT monolayer. 